\title{
MHD flow and heat transfer near the stagnation point on a stretching/shrinking sheet in a micropolar fluid.
}

\begin{abstract}
The problem of two-dimensional magnetohydrodynamic (MHD) stagnation point flow of an incompressible micropolar fluid over a stretching/shrinking sheet is studied. The governing continuity, momentum, angular momentum and heat equations together with the associated boundary conditions are transformed into a system of ordinary differential equations using a similarity transformation. This system is then solved numerically by a finite difference method. The effects of the magnetic parameter, material parameter and the stretching/shrinking parameter on the flow and heat transfer characteristics are thoroughly examined. Different from a stretching sheet, it is found that the solutions for a shrinking sheet are non-unique.
\end{abstract}

Keyword: MHD; Micropolar fluid. 